\title{
Enhanced absorption and electro-optic Pockels effect of electrostatically self-assembled CdSe quantum dots
}

\author{
Fajian Zhang, Liangmin Zhang, You-Xiong Wang, and Richard Claus
}

\begin{abstract}
The spectrum and electro-optic properties of CdSe quantum dots are studied. Spectrum wavelength shifts that are due to the quantum size effect and to the electro-optic Stark effect are investigated. It is found that CdSe quantum dot-polymer composites formed by an electrostatic self-assembly (ESA) technique exhibit high internal electric fields. Using the second-order perturbation theory of the $1 s-1 s$ energy shift (Stark effect), we estimate the internal field of the ESA film to be as high as $2.6 \times 10^{8} \mathrm{~V} / \mathrm{m}$. This value results in a much higher absorption coefficient and electro-optic coefficients for ESA films than for their bulk crystal counterparts or for spin-coated film samples. The relationships among unusual spectra, film structure, and high electro-optic response are analyzed. These results are useful both for understanding the physical mechanisms of semiconductor quantum dots and for developing high-performance photonic devices. (C) 2005 Optical Society of America
\end{abstract}

OCIS codes: $\quad 160.2100,310.6860,300.6170,260.3800$.

\section{Introduction}

Semiconductor nanocrystal quantum dots (SCQDs) have received much attention owing to their unique optoelectronic properties, and they have been used for certain optoelectronic devices such as lasers and detectors. They also exhibit electro-optic and nonlinear optical properties that are different from those of corresponding bulk materials, which themselves hold considerable promise for applications in electronics and photonics, ${ }^{1-4}$ such as electro-optic modulators and optical switches. The optoelectronic properties and applications of SCQDs have been extensively explored, but their electro-optic and nonlinear optical properties and applications, especially the electrooptic Pockels effect and second-order nonlinear [second harmonic generation (SHG)] optical effects, have been scarcely investigated.

In the traditional theory of the origin of SHG in terms of electro-optic coefficients $\left(r_{33}, r_{13}\right)$, the basic structural requirement of materials is the lack of an inversion center. ${ }^{5}$ This is the case, for example, for polymer and crystal electro-optic materials. Because

The authors are with the Fiber \& Electro-Optics Research Center, Virginia Polytechnic Institute and State University, Blacksburg, Virginia 24061. R. Claus's e-mail address is roclaus@vt.edu.

Received 9 April 2004; revised manuscript received 14 February 2005; accepted 17 February 2005.

0003-6935/05/193969-08 $\$ 15.00 / 0$

(C) 2005 Optical Society of America of this restriction, only a small portion of bulk materials exhibit substantial electro-optic properties. For SCQDs, the origin of SHG and Pockels effects is not the lack of an inversion center; instead it is (1) the quantum-confinement effect, (2) surface effects, and (3) defect and trap states. As the sizes of SCQDs are small enough to be comparable with their exciton Bohr radii, quantum-confinement effects predominate. 6,7

There are two major effects that are responsible for these size variations in nanocrystal properties that are caused by changes in state density. First, the intrinsic properties of nanocrystals are determined by the quantum-size effect, which results in quantum confinement and enhanced oscillation strength in compressed excitons. Figure 1 illustrates the enhanced confinement and oscillation strength in such compressed excitons. In bulk crystals, excitons have radius $a_{B}$. In quantum wells (two dimensions) and quantum dots (three dimensions) the size of the material is comparable to or less than $a_{B}$, so excitons are confined to a small region; enhanced electron and hole wave-function overlap results, so the oscillation strengths of the excitons are increased. The second aspect of the size effect is that for nanocrystals the number of surface atoms is a large fraction of the total number of atoms, so a large number of surface states are created. Therefore the resultant dangling orbitals of unpassivated sites on the nanocrystal surface are highly polarizable, and the electron distribution about surface atoms is inherently highly 


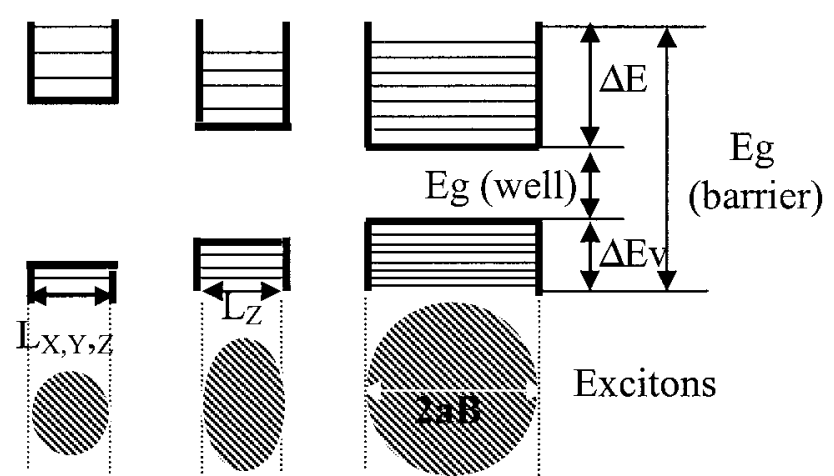

Fig. 1. Enhanced confinement and oscillation strength in compressed excitons.

noncentrosymmetric, which substantially contributes to a second-order nonlinear optical response. ${ }^{6}$

Further, a heavier hole has been predicted theoretically to have an enhanced probability of presence at the surface; this leads to strong electron-hole charge separation in small nanocrystals. ${ }^{6}$ In such a situation, if the stimulation energy is higher than the exciton oscillator energy, excitons will be formed. When they interact with phonons, they will lose some energy. In SCQDs the exciton concentration is higher than in bulk materials. In addition, surface and defect states result in dangling bonds, and trap states cause the separation of electrons and holes. Dielectric mismatch between the cluster and the surrounding matrix (such as a polymer or glass) also produces a dipole moment layer about the clusters, and permanent dipole moments have been observed, ${ }^{7-10}$ so large electro-optic and nonlinear optic effects result. Most research on the electro-optic and nonlinear optic effects of SCQDs is focused on third-harmonic generation and electro-optic Kerr effects, in part because they do not require an asymmetric structure. But, under certain conditions, such as in the presence of an external field, or by introduction of an internal field, central symmetry can be broken, so secondorder electro-optic and incoherent SHG effects (such as the Pockels effects) can be observed.

Electrostatic self-assembly (ESA) is a type of thinfilm fabrication technique; it is based on the electrostatic interaction of anions and cations. Figure 2 shows how films are constructed by this technique.

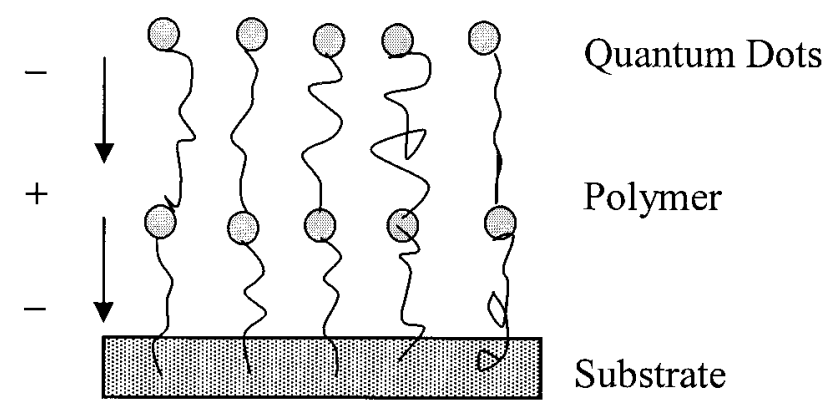

Fig. 2. Structure of a quantum dots-polymer composite film made by ESA.
First the cleaned substrate is charged by chemical processing, so negative surface charges are induced. Then this substrate is dipped into a solution containing polycations, and a monolayer of molecules is attached to the substrate by electrostatic attraction. As this process is repeated, a stack of alternately charged monolayers leads to the creation of an electric field in the films.

Various techniques have been used to produce SCQDs. Patterned arrays of self-assembled SCQDs have been produced on substrates by electron-beam lithography, laser-interference lithography and reactive ion etching, metalorganic molecular-beam epitaxy, rf magnetron cosputtering, and solution synthesis. Highly organized and monodispersed nanoscale patterns result. The Stranski-Krastanow approach to fabricating SCQDs is the most widely used and utilizes the spontaneous islanding phenomena that are due to lattice-mismatch induced strains in epitaxial layers. Many III-V SCQDs such as InGaAs have been fabricated successfully in this way, although researchers generally have not been so successful in fabricating II-VI SCQDs such as CdSe, and especially in utilizing and exploring quantum effects of II-VI SCQDs in the strong confinement region. This region has been sparsely investigated because of difficulty in the synthesis of small-sized, high-quality SCQDs and because the exciton Bohr radius of CdSe is very small $(\sim 5.4 \mathrm{~nm}) .{ }^{11}$

In our previous research we demonstrated that ESA electro-optic films are quite different from traditional films in that they have high electro-optic Pockels coefficients and because no poling voltage is needed, and they have anisotropic structure. ${ }^{12,13}$ But the reasons for these differences were not known. Our purpose in this paper is to use spectroscopy technology to investigate the characteristics of such materials and find the reasons for such novel characteristics. As is well known, for electro-optic materials an external poling electric field is usually needed in experiments for the measurement of electro-optic coefficients. For the first time to our knowledge we have demonstrated that a strong electro-optic Pockels effect can be achieved for ESA electro-optic films in the absence of external electric fields. In this case it is expected that local electric fields will be essential for electro-optic Pockels and SHG effects. Such local electric fields can be generated by factors such as stress and dislocations. Here we propose that ESA techniques are the best generators of local electric fields. Based on absorption and photoluminescence spectra of ESA and spin-coated samples, we analyze the quantum-size effect and the electro-optic Stark effect and use the results of the analysis to explain the resultant unusual structures and electro-optic properties of SCQDs and to support our conclusions.

\section{Experiment}

CdSe quantum dots were prepared by a metalorganic high-temperature solution method. Dimethyl cadmium $\left[\mathrm{Cd}\left(\mathrm{CH}_{3}\right)_{2}\right]$ and bis(trimethylsilyl) sulfide 
$\left[(\mathrm{TMSi})_{2} \mathrm{Se}\right]$ were dissolved in trioctyphosphine $\left[\left(\mathrm{CH}_{3}\left(\mathrm{CH}_{2}\right)_{7}\right)_{3} \mathrm{P}\right]$ in a nitrogen saturated dry box. They were mixed together at room temperature and then injected into preheated trioctyphosphine oxide $\left[\left(\mathrm{CH}_{3}\left(\mathrm{CH}_{2}\right)_{7}\right)_{3} \mathrm{PO}\right.$; TOPO $]$. The cadmium and selenium organometallic precursors were immediately deposited at high temperatures $\left(300^{\circ}-350^{\circ} \mathrm{C}\right)$ to form CdSe as seeds, and the solvent TOPO served as a capping agent. We monitored the reaction by measuring the absorption spectrum or the luminescence spectrum of the reaction solution regularly. Different particle sizes correspond to different peak positions of the absorption and luminescence spectra. One can obtain an approximate estimation of the particle size simply by watching the color of the solution. The surfaces of the CdSe quantum dots were modified and the resultant functionalized quantum dots were dispersed in a polymer host by the ESA technique for various measurements by methods described elsewhere. ${ }^{12,13}$ For comparison, SCQD-doped polymeric electro-optic films were also fabricated by spin coating. Nanoparticles were mixed with Norland optical glue at a concentration of $0.5 \%-5 \%$ and these films were cured with UV light at a wavelength of $365 \mathrm{~nm}$ for $5 \mathrm{~min}$. The films were poled at an approximate field strength of $8 \times 10^{7} \mathrm{~V} / \mathrm{m}$ at an elevated temperature $\left(>90^{\circ} \mathrm{C}\right)$ for $60 \mathrm{~min}$.

Optical absorption and photoluminescence of fabricated SCQDs and electro-optic films were characterized by UV-visible absorption spectra (with a U-2001 spectrophotometer from Hitachi Instruments, Inc.; scan speed, $400 \mathrm{~nm} / \mathrm{min}$ ) and by photoluminescence spectra (with an F-4500 fluorescence spectrophotometer from Hitachi Instruments). Film thickness was measured with ellipsometers (a Rudolph/Auto EL at fixed wavelength $632 \mathrm{~nm}$ and a Woollam VB-200 at variable wavelength 200$1030 \mathrm{~nm}$ ). Images were obtained by use of a highresolution transmission-electron microscope (Philips$420 \mathrm{~T}$ electron microscope operating at $100 \mathrm{kV}$ ). These images were obtained under bright-field conditions. We prepared cluster samples by first dispersing nanoclusters in toluene and then placing a drop of the dilute dispersion of nanoclusters on the surfaces of carbon-coated copper grids. Electro-optic coefficient measurements are described elsewhere. ${ }^{12,13}$

\section{Results and Discussion}

\section{A. Spectrum Analyses}

To compare the particle size results obtained from absorption spectra, we used transmission-electron microscopy to obtain images of CdSe quantum dots, as shown in Fig. 3. From a transmission-electron microscope image of the CdSe particles we found the particle sizes (diameters) to be approximately 2-3 nm, with narrow size distribution. The sizes are small compared with the exciton Bohr radius, so strong quantum confinement is expected.

A change in radius of the nanoclusters leads to a wavelength shift in absorption and luminescence spectra, and from this information the particle size

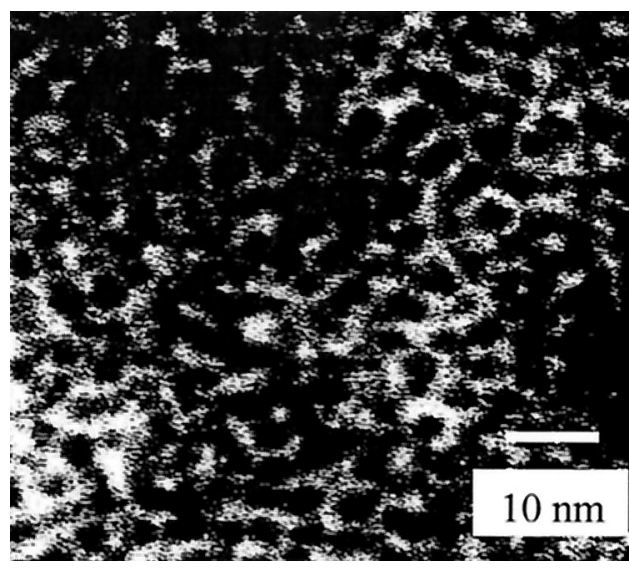

Fig. 3. Transmission-electron microscope image of CdSe quantum dots.

can be calculated. ${ }^{14}$ This size effect can be analyzed based on the Schrödinger equation in quantummechanical theory, for which the exciton can be described by the Hamiltonian ${ }^{15}$

$$
\hat{H}=-\frac{h^{2}}{2 m_{h}} \nabla_{h}^{2}-\frac{h^{2}}{2 m_{e}} \nabla_{e}^{2}-\frac{e^{2}}{\varepsilon\left|r_{e}-r_{h}\right|},
$$

where $m_{h}$ and $m_{e}$ are the effective masses of holes and electrons, respectively, $\varepsilon$ is the dielectric constant, and Hamiltonian, kinetic, Coulomb, and polarization terms are included. The polarization term can be ignored because it is small in comparison with kinetic and Coulombic interactions. The wave function of the excitons can be found, so the energy state of the excitons can be determined. With the wave function, the energy of the lowest excited states becomes ${ }^{11}$

$$
E(R)=E_{g}+\frac{h^{2} \pi^{2}}{2 \mu R^{2}}-\frac{1.786 e^{2}}{\varepsilon_{2} R}+0.248 E_{R},
$$

where

$$
\begin{aligned}
E_{R} & =\frac{13.606 m_{0}}{\varepsilon_{2}^{2}}\left(\frac{1}{m_{e}{ }^{*}}+\frac{1}{m_{h}{ }^{*}}\right), \\
\frac{1}{\mu} & =\frac{1}{m_{e}{ }^{*}}+\frac{1}{m_{h}{ }^{*}},
\end{aligned}
$$

$m_{e}{ }^{*}$ and $m_{h}{ }^{*}$ are electron and hole effective masses, respectively, and $\mu$ is the reduced exciton mass. Equation (2) also describes the effect that the band edge of the absorption spectrum varies with particle size. In Eq. (2), the first term is the bulk energy gap. The second term is the kinetic term that corresponds to quantum-localization energy because of the quantum-confinement effect and is proportional to $1 / R^{2}$. The third term is the Coulombic term proportional to $1 / R$, and the last term is the correlation energy. As the particle size is small, the kinetic term predominates. 


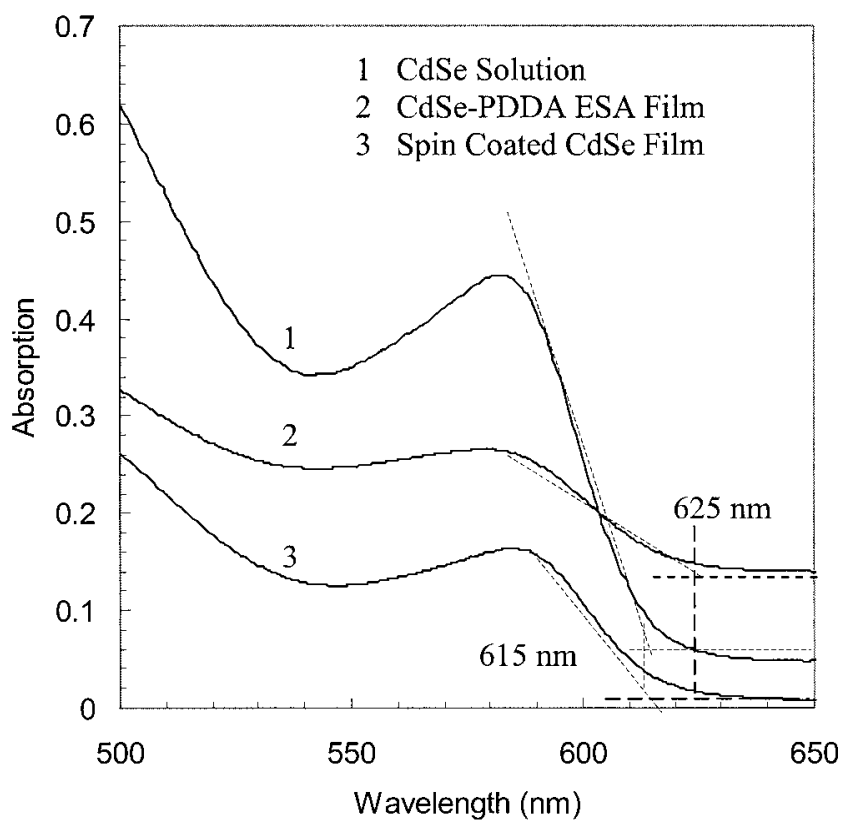

Fig. 4. Optical absorption spectra of CdSe films and solution (concentrations of CdSe quantum dots: ESA film, 2.14\%; spincoated film 5\%). PDDA, poly(diallyldimethylammonium chloride)].

Figure 4 shows the absorption spectra of the CdSe solution and films. Note that the band edge of the lowest $1 s-1 s$ state is apparently shifted from the nominal value of $716 \mathrm{~nm}$ for bulk CdSe (at room temperature, $15 \mathrm{~K}$, it is $680 \mathrm{~nm})^{16}$ to $615 \mathrm{~nm}$ for a solution and spin-coated film and to $625 \mathrm{~nm}$ for ESA film. There is a $10 \mathrm{~nm}(32.3 \mathrm{meV})$ shift between the solution-spin-coated film and the ESA film.

Figure 5 shows the luminescence spectra of the same samples. From the luminescence spectra, the threshold wavelengths of the ESA and spin-coating films are 625 and $615 \mathrm{~nm}$, respectively, the same as the results for the absorption spectra. The photon energy difference at peak intensity is $46.9 \mathrm{meV}$ $(13 \mathrm{~nm})$, and the FWHM is $131 \mathrm{meV}$ for the ESA film and $135 \mathrm{meV}$ for the spin-coated film. This means a

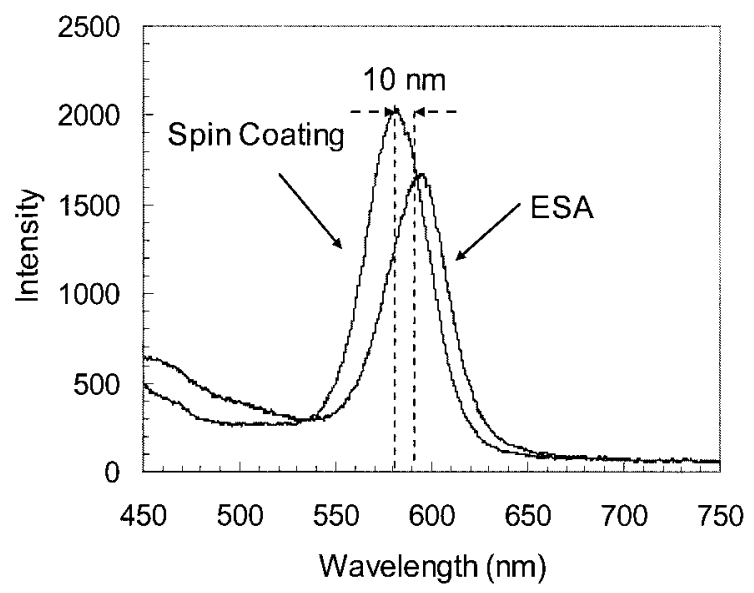

Fig. 5. Luminescence spectra of CdSe films prepared by spin coating and ESA.

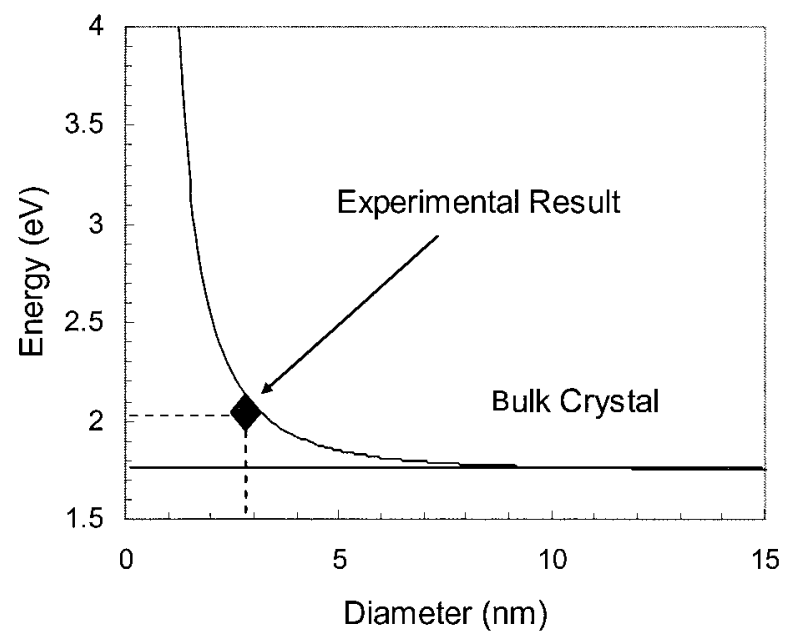

Fig. 6. Experimental and theoretical results of $1 s-1 s$ transition energy of CdSe crystallites as a function of size.

fairly narrow size distribution and good uniformity. The peak positions of these spectra are quite close to those of the absorption spectra.

A spectrum shift can be induced by the quantumsize effect (blueshift) and the field-induced electrooptic Stark effect (redshift), and for the shift between bulk CdSe and a CdSe solution it is reasonable to assume that the blueshift occurs exclusively. For CdSe, $E_{g}=1.70 \mathrm{eV}(300 \mathrm{~K}), \varepsilon_{2}=9.4,{ }^{10} m_{e}$ $=0.13 m_{0}, m_{h}=0.45 m_{0},{ }^{17}$ and, based on Eq. (2), the variation in absorption band-edge energy and wavelength shift relative to the particle size of the CdS quantum dots can be determined. The particle size can thus be calculated. Calculated and measured spectral shifts are plotted in Fig. 6. Comparing this calculation result with absorption and TEM measurement results, we can find that, at the same particle size, the actual shift is $5 \%$ smaller than the calculated shift, because this model overestimates the shift for smaller clusters. The discrepancy means that this is a simple model and that more factors should be considered, but a 5\% discrepancy is small.

\section{B. Internal Electric Field Calculation}

From the absorption and luminescence spectra, there is another shift between the solution-spin-coated film and the ESA film. This shift cannot be explained by quantum-size effects because, in the experiment, the same batch of quantum dots was used, so the particle size is the same for the solution and the films. Thus, if there is some size difference, the absorption threshold and peak for ESA films should occur at higher energy (blueshift) because the ESA film was made in a water solution, and size selection may apply automatically owing to the precipitation of larger particles. From Figs. 4 and Fig. 5, however, it is apparent this does not happen. Instead, the ESA film has a lower absorption threshold and absorption-luminescence peak position (redshift). ESA and spin-coating films were prepared and treated without stress, so we suspect that this shift is 
due mainly to the Stark effect or to the field-induced $1 \mathrm{~s}-1 s$ energy shift, although some unknown factors may also be present. The Stark effect is also based on the quantum-mechanical theory of wave functions, and an analysis of several kinds of interaction, as discussed above, reveals that the shift in optical absorption originates from another factor, namely, the external or internal electrical field. In the analysis, the simplified model ignores Coulomb interaction and the anisotropic parts of the whole Hamiltonian. As field $F$ is applied, some oscillator strength is redistributed into transitions that are blocked for the nonfield case. The second-order perturbation theory may then be applied, leading to the shift ${ }^{18}$

$$
\Delta E(F)=\frac{-0.65(e F R)^{2}}{E_{1 s}},
$$

where

$$
E_{1 s}=\frac{h^{2} \pi^{2}}{2 R^{2}}\left(\frac{1}{m_{e}}+\frac{1}{m_{h}}\right)
$$

and $R$ is the radius of the particles. From the theory, shift $\Delta E$ is proportional to $R^{4}$ and $F^{2} . F$ can be either an external applied field or an internal field. For the Stark effect that is due to molecules or a polymer, $F$ is an internal field because of a number of factors such as the ESA process or fabrication of the film under the influence of an external field (field-assisted ESA).

From Eqs. (5) and (6), $F$ can be determined by shift $\Delta E$ if radius $R$ of the SCQDs is known. Taking the average $R=1.25 \mathrm{~nm}$, we find from the absorption spectra that a $10 \mathrm{~nm}$ threshold wavelength shift is clearly shown, which corresponds to a $1 s-1 s$ energy shift $\Delta E$ of the ESA film with respect to the spincoated film, or that the CdSe solution is $-32.3 \mathrm{meV}$. We thus can estimate that the internal field $(F)$ of the ESA film is $2.6 \times 10^{8} \mathrm{~V} / \mathrm{m}$; from the luminescence spectra, the result is the same. This field is high, we suspect that the resultant unusual structures and novel electro-optic properties in our previous experiments stem from the internal field, which makes ESA films remarkably different from spin-coated films. The consequences of the high internal field are demonstrated and discussed below.

\section{Unique Properties of ESA Films Owing to Their Internal Fields}

\section{Absorption Coefficient}

In addition to the shift between samples in Fig. 4, the absorption coefficients of ESA films are found to be much higher than those of spin coated films. From Fig. 4, the absorption of a CdSe-PDDA film made by ESA processing is only slightly larger than that of a spin-coated CdSe film. From x-ray photoemission spectra of the ESA films, the calculated concentration of CdSe quantum dots in the sample is $2.14 \%, 11$

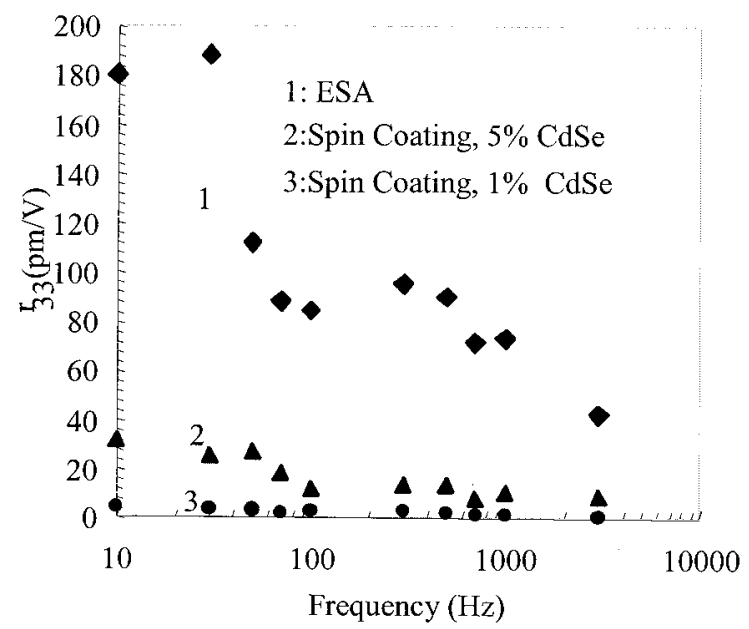

Fig. 7. Variation of $r_{33}$ with modulation frequency of CdSe films fabricated under different conditions. The poling voltage of the spin-coating film is $80 \mathrm{~V} / \mu \mathrm{m}$.

which is very close to the concentration of CdSe quantum dots in spin-coated samples (5\%). The structure of the ESA film is shown in Fig. 2. The film has 24 bilayers, film growth is uniform, each bilayer has almost the same thickness $(6 \mathrm{~nm})$, the total thickness is $144 \mathrm{~nm}$, and the thickness of spin-coated CdSe-PDDA film is $76 \mu \mathrm{m}$. With the film thickness taken into account, the ratio of absorption coefficient $\alpha$ of ESA films to that of spin-coated films is 2.1 $\times 10^{3}$. This is one of the most important differences between spin coating and ESA processing for film fabrication.

\section{Electro-Optic Effect}

Figure 7 shows how $r_{33}$ varies with the modulation frequency of CdSe films fabricated under different conditions. From this figure we conclude that $r_{33}$ decreases with increasing modulation frequency; that the $r_{33}$ of spin-coated films varies with SCQD concentration; most importantly that ESA films have higher $r_{33}$ than spin-coated films; and that no poling voltage is needed. Like the absorption coefficient result in Subsection 3.C.1, this is an unusual result. For general conditions (normal temperature and pressure), CdSe nanocrystals belong to the hexagonal (wurtzite) system and have a $6 \mathrm{~mm}$ symmetry structure, and the crystals' electro-optic coefficients are $r_{33}$ $=4.3(\mathrm{pm} / \mathrm{V})$ and $r_{13}=1.8(\mathrm{pm} / \mathrm{V}){ }^{19}$ It is interesting that, compared with the experimental results, here the CdSe SCQDs have much higher electro-optic coefficients than their bulk crystal counterparts. This is so because they have totally different origins of their electro-optic effects. Lack of an inversion center in the hexagonal crystal class is the basis of the electro-optic effect of bulk CdSe crystal, although the electro-optic effect is small, like that most of other crystals. The electro-optic effect of SCQDs is further enhanced by ESA processing.

As mentioned above, an external electric field is usually needed for the measurement of electro-optic coefficients of electro-optic materials; for the first 


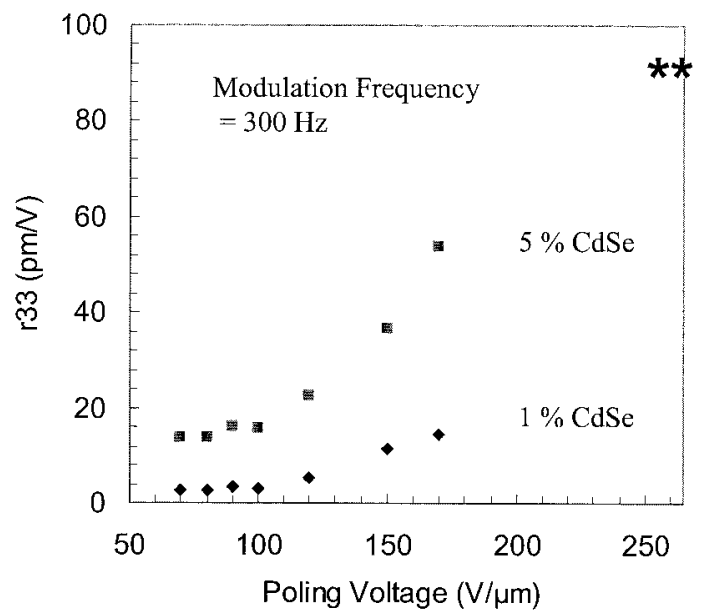

Fig. 8. Variation of $r_{33}$ with poling voltage of spin-coating CdSe films. The modulation frequency is $300 \mathrm{~Hz}$, and the double asterisks mark $r_{33}$ of the ESA film obtained from Fig. 7 .

time to our knowledge we demonstrate that a high electro-optic Pockels effect can be achieved for ESA electro-optic films in the absence of external electric fields. This result can now be explained as the effect of a local or built-in electric field that is introduced by ESA techniques. For spin-coated films, a poling field of $8 \times 10^{7}-12 \times 10^{7} \mathrm{~V} / \mathrm{m}$ is needed to achieve an apparent electro-optic effect. In Fig. 7 the poling voltage of the spin-coating film is $8 \times 10^{7} \mathrm{~V} / \mathrm{m}$. Higher voltage results a higher electro-optic coefficient but, in practice, the voltage cannot be too high; otherwise the films may be damaged. The highest voltage that can be reached in our experiment is $\sim 17 \times 10^{7} \mathrm{~V} / \mathrm{m}$. At this voltage the electro-optic coefficients of spin-coating films are much higher than the electro-optic coefficient obtained at 8 $\times 10^{7} \mathrm{~V} / \mathrm{m}$. Figure 8 shows the experimental variance of $r_{33}$ with the poling voltage for spin coating CdSe films at a modulation frequency of $300 \mathrm{~Hz}$ : The poling voltage is as much as $17 \times 10^{7} \mathrm{~V} / \mathrm{m}$. In this figure the double asterisk stands for $r_{33}$ of the ESA film shown in Fig. 7. From Fig. 8 it is expected that, if the poling field can reach the calculated internal field of ESA films, the electro-optic coefficients of spin-coated films can approach those of ESA films. This means that the amplitude of the calculated internal field in an ESA is reasonable and supports the assumption that the wavelength shifts in Figs. 5 and Fig. 6 are due mainly to the electro-optic Stark effect. Because this high field helps to align the dipole moments of the polymers and semiconductor quantum dots, noncentrosymmetric structures and large dipole moments can result, thus giving rise to large electro-optic responses.

\section{Anisotropy Structure}

The third unusual result of the high internal field in ESA films is film-structure anisotropy. From symmetry considerations, the value of $r_{333} / r_{113}$ of isotropic electro-optic materials should be $3 .{ }^{20}$ It is assumed that a poled glassy chromophore thin film is uniaxial and belongs to the $C_{\infty}$ point group, so $r_{13}=r_{51}$. If there is no interaction between chromophores, based on a standard rigid gas model, one can predict that $r_{33}=3 r_{13}$. This conclusion has long been used for polymeric electro-optic materials, but, for many organic electro-optic materials such as LangmuirBlodgett films, liquid crystals, and organic crystals, which display a fair degree of anisotropy, $r_{33} / r_{13}$ $\neq 3$. Our previous research showed that the ratio for ESA films varies from 4.1 to 4.5 at different modulating frequencies. ${ }^{12}$ Other samples give the same result: $r_{333} / r_{113}>3$. This means that ESA films cannot be treated as an ideal isotropic system with $C_{\infty v}$ symmetry; they are indeed anisotropic. A modified new relationship has been derived, ${ }^{21}$ in which the extent of anisotropy (dispersion factor) is considered.

\section{Theoretical Analysis of Absorption and the Electro-Optic Effect}

In summary, high absorption, high electro-optic effect, and anisotropic structure are found in ESA films. They all have high internal fields, and their relationship can be further explained as follows.

The absorption and electro-optic effects of SCQDs are both based on the oscillation of excitons. An exciton can be seen as an oscillator with a certain special oscillation frequency, and the oscillator strength of an exciton can be expressed as ${ }^{11}$

$$
f=\frac{2 m}{h^{2}} \Delta E|\mu|^{2}\left|u_{0}\right|^{2},
$$

where $m$ is the mass of an electron, $\Delta E$ is the band energy, $\mu$ is the exciting energy, and $u_{0}$ is an electronhole wave function. Here $\left|\mu_{0}\right|^{2}$ is the overlap factor, or the probability of finding electrons and holes at certain locations. As particle size decreases, electrons and holes are confined to a smaller space, so $\left|\mu_{0}\right|^{2}$ increases, and so does the oscillator strength of the excitons. The oscillator strength density is $f / V(V$ is the volume of SCQDs), which determines the absorption coefficients of materials. As particle size decreases, $f$ increases and $V$ decreases, so $f / V$ increases significantly. So does the absorption coefficient of the SCQDs.

$\chi^{(2)}$ varies with orientation angle $\mathrm{as}^{22}$

$$
\chi^{(2)}=N \beta\left[f^{(\omega)}\right]^{2} f^{2 \omega}\left(\cos ^{3} \varphi\right),
$$

where $\beta$ is the second-order susceptibility of the SCQDs or chromophores; $N$ is the number of SCQDs or chromophores per unit volume; $f^{(\omega)}$ and $f^{(2 \omega)}$ are the local field factors, which are simple functions of the refractive index at the fundamental frequency and the double frequency (SHG); $\varphi$ is the average angle between the ground-state dipole moment of the SCQDs or chromophores and the direction normal to the films; and $\cos ^{3} \varphi$ is an acentric order parameter. To have a nonzero Pockels effect, such spin-coated films must be poled before measurement or an elec- 
tric field must be applied during measurement. Experimentally, for ESA electro-optic films a high electro-optic coefficient can be measured in the absence of an external electrical field; this means that an internal electrical field is established.

Some theories have been developed for determining the relationship between optical absorption and the second-order nonlinear dielectric susceptibility, namely, ${ }^{11}$

$$
\chi^{(2)}=\frac{n c}{4 \pi \omega} \alpha
$$

where $n$ is the refractive index, $c$ is the speed of light in vacuum, and $\omega$ is the angular frequency. Based on four-wave mixing theory and by application of Fermi-Dirac statistics of band filling, third-order susceptibility $\chi^{(3)}$ is also proportional to the absorption coefficient. ${ }^{23}$ The electro-optic coefficient is then proportional to the nonlinear optic coefficient. The coefficient is $2 / n^{4}$, because both effects have the same physical origin. Thus ${ }^{24}$

$$
r=2 \chi^{(2)} / n^{4} .
$$

This means that smaller SCQDs result in higher absorption and cause larger nonlinear optical and electro-optic responses. It is thus expected that ESA films will have higher electro-optic coefficients than spin-coated films, provided that their quantum dot concentrations are similar. This assumption is easy to understand because electro-optic and nonlinear optic effects, optical absorption, and other optical properties of materials are determined by radiationatomic system interaction, and the properties of oscillators (oscillation strength and frequency) have a major influence on the results of interaction. The basic nonlinear optical process can be viewed as the absorption of photons in materials. As for SHG, the process involves the annihilation of two photons at frequency $\omega$ and the simultaneous creation of a photon at $2 \omega$. The conversion efficiency is dependent on phase-matching conditions. ${ }^{19}$

By taking advantage of the Kramers-Kronig theory in the study of the electro-optic effect of quantumwell structures, we have also predicted and observed a strong electro-optic effect. Quantum-well structure is similar to that of multilayer films made by the ESA technique. For example, for a GaAs $(6 \mathrm{~nm}) /$ $\mathrm{Ga}_{0.6} \mathrm{Al}_{0.4} \mathrm{As}(10 \mathrm{~nm})$ quantum-well structure, and for an electric field of $80 \mathrm{kV} / \mathrm{cm}$, using the KramersKronig theory yields a calculated maximum variation of refractive index of $\Delta n=1.8 \%$, which is 2 orders of magnitude greater than the refractive-index variation in its bulk crystals. ${ }^{25}$ This result is consistent with experimental results. The enhanced electrooptic effect originates from the enhanced quantum confinement in a quantum-well structure (enhanced two-dimensional confinement). By comparison, it is not difficult to predict the enhanced electro-optic effect in nanocluster-based films (enhanced three- dimensional confinement) and ESA films (enhanced two-dimensional confinement).

\section{Conclusions}

The quantum-size effect and the electro-optic Stark shift of CdSe semiconductor quantum dots have been investigated. Quantum dots were dispersed in a polymer host by both electrostatic self-assembly (ESA) and spin-coating techniques. The unique structure and properties of the ESA films were explored. We suspect that the observed shifts in absorption and luminescence spectra are due mainly to the Stark effect or to a field-induced 1s-1s energy shift. Based on the Stark effect, from spectral analysis this built-in electric field is estimated as $2.6 \times 10^{8} \mathrm{~V} / \mathrm{m}$, much greater than the usual poling fields applied to spin-coated polymeric electro-optic films. We also propose that this internal electric field is the underlying cause of the unusual structure and properties of ESA films presented here, which exhibit much stronger absorption than spin-coated films and have much higher electro-optic coefficients than their bulk crystal counterparts and spin-coated films. More importantly, for ESA films no poling voltage is needed for alignment and a high electro-optic effect to be achieved. The electric field makes ESA films have totally different origins in their electro-optic effects, and ESA films cannot be treated as an ideal isotropic system with $C_{\infty<}$ symmetry. Experimental results match the theoretical calculation well and support our conclusions.

This study was supported in part by the U.S. Army Multidisciplinary University Research Initiative program (DAAD19-02-1-0275) and in part by the Optical Science and Engineering Research Center of Virginia Polytechnic Institute. The authors thank William B. Spillman, Jr., and Ken E. Meissner for technical assistance.

\section{References}

1. A. P. Alivisatos, "Perspectives on the physical chemistry of semiconductor nanocrystals," J. Phys. Chem. 100, 13,22613,239 (1996).

2. T. D. Krauss, F. W. Wise, and D. B. Tanner, "Observation of coupled vibrational modes of a semiconductor nanocrystal," Phys. Rev. Lett. 76, 1376-1379 (1996).

3. M. V. Artemyev and U. Woggon, "Quantum dots in photonic dots," Appl. Phys. Lett. 76, 1353-1355 (2000).

4. T. Trindade, P. O. Brien, and N. L. Pickett, "Nanocrystalline semiconductors: synthesis, properties, and perspectives," Chem. Mater. 13, 3843-3858 (2001).

5. A. Yariv, Optical Electronics in Modern Communications, 5th ed. (Oxford U. Press, 1997), p. 327.

6. M. Jacobsohn and U. Banin, "Size dependence of second harmonic generation in CdSe nanocrystal quantum dots," J. Phys. Chem. B 104, 1-5 (2000).

7. S. A. Blanton, R. L. Leheny, and M. A. Guyot-Sionnest, "Dielectric dispersion measurements of CdSe nanocrystal colloids: observation of a permanent dipole moment," Phys. Rev. Lett. 79, 865-868 (1997).

8. M. P. Halsall, J. E. Nicholls, J. J. Davies, P. J. Wright, and B. Cockayne, "Photoluminescence studies of CdS, CdSe wurtzite 
superlattices; evidence for large piezoelectric effects," Surf. Sci. 228, 41-44 (1990).

9. S. A. Empedocles and M. G. Bawendi, "Quantum-confined Stark effect in single CdSe nanocrystallite quantum dots," Science 278, 2114-2117 (1997).

10. V. L. Colvin and A. P. Alivisatos, "CdSe nano-crystals with a dipole moment in the first excited state," J. Chem. Phys. 97, 730-733 (1992).

11. A. D. Yoffe, "Low-dimensional systems: quantum size effects and electronic properties of semiconductor microcrystallites (zero-dimensional systems) and some quasi-two-dimensional systems," Adv. Phys. 42, 173-266 (1993).

12. L. M. Zhang, F. J. Zhang, Y. Q. Wang, and R. O. Claus, "Linear electro-optic tensor ratio determination and quadratic electrooptic modulation of electrostatically self-assembled CdSe quantum dot films," J. Chem. Phys. 116, 6297-6304 (2002).

13. L. M. Zhang, F. J. Zhang, K. Cooper, Y. Q. Wang, Y. J. Liu, and R. O. Claus, "Electro-optic property measurements of electrostatically self-assembled ultrathin films," Opt. Commun. 186, 135-141 (2000).

14. F. Zhang, "Electro-optic properties of semiconductor nanocrystals and electro-optic polymers and their applications," Ph.D. dissertation (Virginia Polytechnic Institute, 2002).

15. L. E. Brus, "Electron-electron and electron-hole interactions in small semiconductor crystallites: the size dependence of the lowest excited electronic state," J. Chem. Phys. 80, 4403-4409 (1984).
16. C. B. Murray, D. J. Norris, and M. G. Bawendi, "Synthesis and characterization of nearly monodisperse $\mathrm{CdE}(E=\mathrm{S}$, Se, Te) semiconductor nano-crystallites," J. Am. Chem. Soc. 115, 8706-8715 (1993).

17. S. M. Sze, Physics of Semiconductor Devices, 2nd ed. (Wiley, 1981).

18. L. E. Brus, "Quantum crystallites and nonlinear optics," Appl. Phys. A 53, 465-474 (1991).

19. A. Yariv, Optical Electronics in Modern Communications, 5th ed. (Oxford U. Press, 1997), pp. 334-335.

20. C. C. Teng and H. T. Man, "Simple reflection technique for measuring the electro-optic coefficient of poled polymers," Appl. Phys. Lett. 56, 1734-1736 (1990).

21. G. A. Lindsay, Polymers for Second-Order Nonlinear Optics (American Chemical Society, 1995).

22. M. A. Mortazavi, A. Knoesen, and S. T. Kowel, "Secondharmonic generation and absorption studies of polymer-dye films oriented by corona-onset poling at elevated temperatures," J. Opt. Soc. Am. B 6, 733-741 (1989).

23. R. C. Caro and M. C. Gover, "Phase conjugation by degenerate four-wave mixing absorbing media," IEEE J. Quantum Electron. 18, 1376-1380 (1982).

24. S. M. Amsterdam, Nonlinear Optics: Fundamentals, Materials and Devices: Proceedings of the Fifth Toyota Conference on Nonlinear Optical Materials (North-Holland, 1992), p. 421.

25. R. G. Hunsperger, Integrated Optics Theory and Technology, 4th ed. (Springer-Verlag, 1995). 